\title{
High-frequency variations of hydrogen spectral lines in the B3V star $\eta \mathrm{UMa}$
}

\author{
S. M. Pokhvala* \\ Main Astronomical Observatory of NAS of Ukraine, Akademika Zabolotnoho str., 27, 03680 Kyiv, Ukraine
}

\begin{abstract}
We reported the detection of high-frequency variations in the hydrogen Balmer lines in the hot star $\eta$ UMa of spectral class B3V. Spectral observations of $\eta$ UMa were carried out with slitless spectrograph $(\mathrm{R} \sim 100)$ installed on the $60 \mathrm{~cm}$ Carl Zeiss telescope in the Andrushivka Observatory. Spectra were obtained with a time resolution in the sub-second range. It has been found that the $\eta \mathrm{UMa}$ shows rapid variations in the hydrogen lines $\mathrm{H} \alpha, \mathrm{H} \beta$, $\mathrm{H} \gamma$, as well as variations in the atmospheric oxygen lines. The intensity variations in the hydrogen lines varies from $0.2 \%$ to $0.5 \%$, and that of the oxygen lines is approximately $2 \%$.
\end{abstract}

Key words: instrumentation: detectors, methods: observational, techniques: image, processing techniques: spectrometric, stars: imaging

\section{INTRODUCTION}

The stars of spectral type B are high-luminosity blue stars. Their spectra contain lines of neutral helium, with maximum intensity of subclass B2. Their spectra contain hydrogen lines, as well as lines of ionized metals including MgII and Si. The B-type stars often reveal variations in the line HeII (1640 $\AA$ ) [1], as well as emission in the infrared region [2]. $\eta$ UMa is a main-sequence star of the spectral class B3V, with mass $6 \mathfrak{M}_{\odot}$ and radius $1.8 \mathfrak{R}_{\odot}$, an effective temperature of $22000 \mathrm{~K}$. In 1951 the star was added to variables Catalogue of suspected variable stars [3]. Cassinelli [4] had studied the data from the cosmic ray observatory ROSAT, to detect the emission lines in B stars. His study did not confirm the presence of emission from $\eta$ UMa. B-type stars do not have coronae; they do not have a highly convective zone in the upper atmosphere, as is the case with chromospherically active stars of late spectral types. However, they often display a high rate of mass loss and stellar winds of speeds up to $3000 \mathrm{~km} / \mathrm{s}$. All of these factors give some ground to search for both photometric and spectral variability in B-type stars. In this paper we present evidence of spectral variability of $\eta \mathrm{UMa}$ with amplitudes of tenths of a percent in the sub-second range.

\section{OBSERVATIONS}

Spectral observations of $\eta \mathrm{UMa}$ were carried in July 2014 in the Andrushivka Astronomical Observatory. The goal of the observations was to obtain spectra of hot stars (the divisors), in order to calibrate spectra of program stars. However, while pro-

\footnotetext{
*nightspirit10@gmail.com
}

(C) S. M. Pokhvala, 2015 cessing the observations, we found that $\eta \mathrm{UMa}$ shows fast variation in the hydrogen lines. Such variations are mainly typical for late-type stars with chromospheric activity. To investigate the spectral variability of $\eta$ UMa 200 spectra were obtained with an exposure of 0.1 seconds and a time resolution of 1.96 seconds. Fig. 1 shows recorded spectra of $\eta$ UMa.

To verify the presence of variability it is necessary to show that such a variability is absent in a comparison star. For this purpose, we used the comparison star $77 \mathrm{Cyg}$ of spectral type A0V, V = 5.73. Fig. 2 shows the Ca II H, K and hydrogen absorption lines from a sample of 500 spectra with a time resolution 2.88 seconds in 77 Cyg. Fig. 3 shows the relative variation in the spectrum of $77 \mathrm{Cyg}$, equal to the ratio of the standard deviations of spectra to the average intensity in the spectrum at a given wavelength. In the absence of variability the minima of relative variations coincide with the minima of intensities. This is due to the fact that in random variables with Poisson distribution, the mean and the variance are equal. Thus, Figs. 2, 3 suggest that variations in the spectrum of $77 \mathrm{Cyg}$ are absent.

To eliminate continuous spectrum of a star (continuum) and obtain the absorption spectra we used high-frequency filtering of spectra with the Kaiser digital filter. Details of filtering techniques can be found in $[5,7]$. The transmission curves of the digital filter are shown in Fig.4 The lower limit of the filter corresponds to the spatial frequency of $1 / 50$ pixel $^{-1}$, the top of $1 / 30$ pixel $^{-1}$. One pixel corresponds to $9 \AA$ in the wavelength scale. The filter suppresses a signal in the stopband frequency up to 40 decibels. 


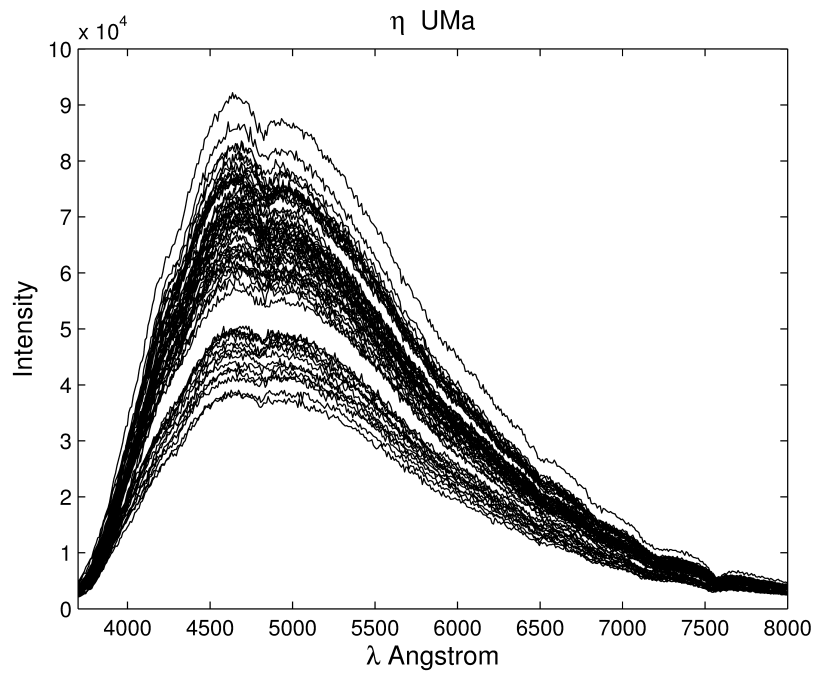

Fig. 1: Low-resolution slitless spectra of $\eta \mathrm{UMa}$.



Fig. 2: Averaged absorption spectra of $77 \mathrm{Cyg}$.

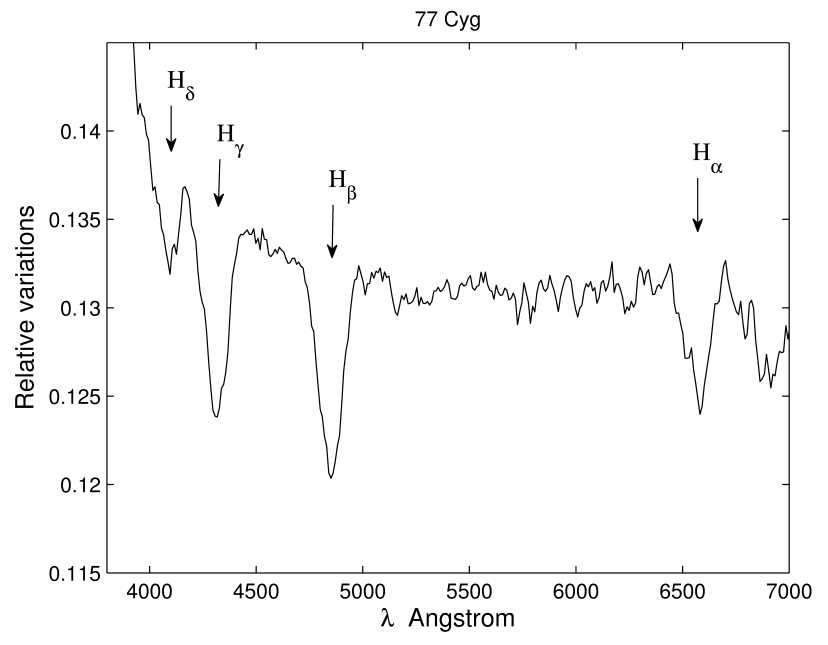

Fig. 3: Variations in spectrum of $77 \mathrm{Cyg}$.
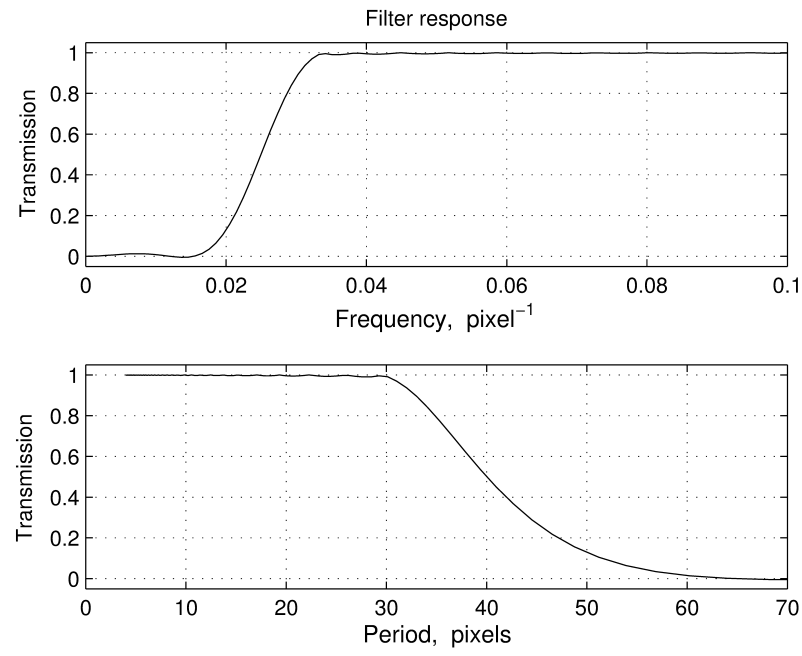

Fig. 4: Transmission curve of the Kaiser filter.
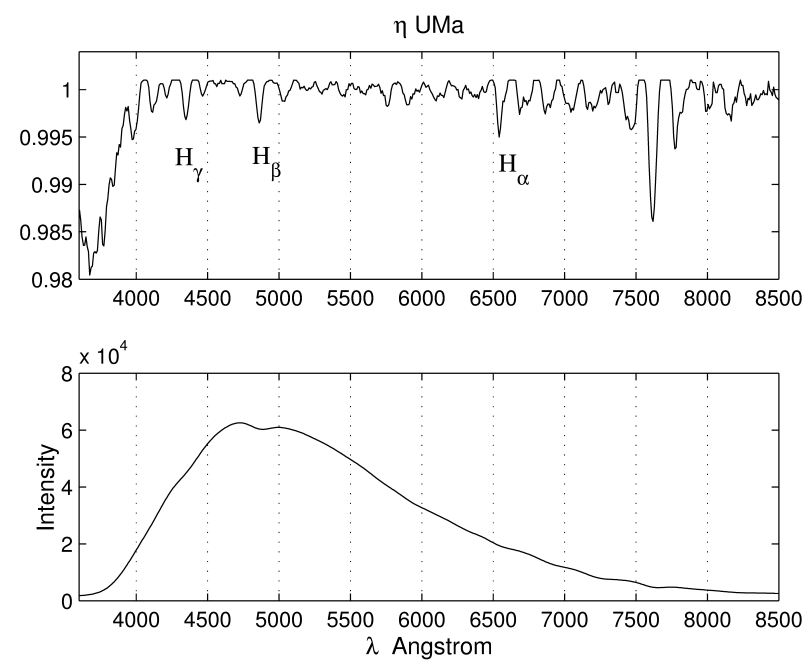

Fig. 5: Averaged absorption spectra (upper) and continuum (bottom).

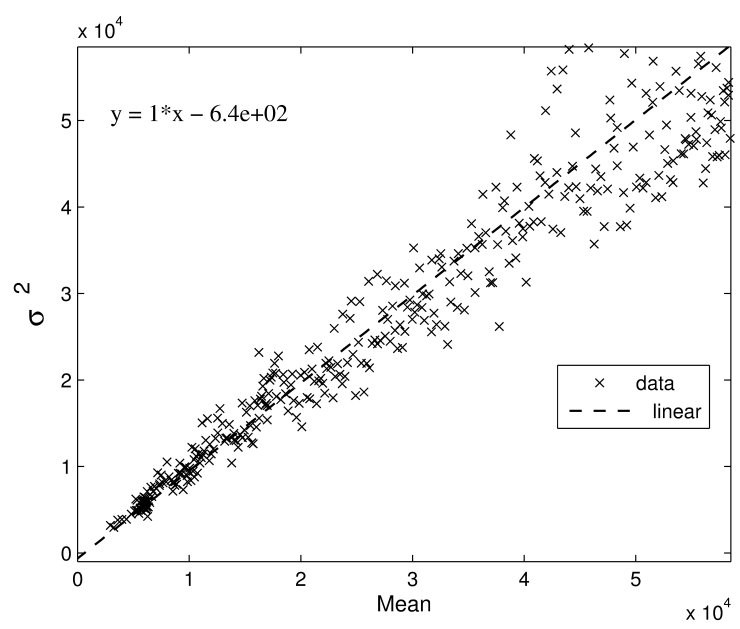

Fig. 6: $\sigma^{2}$ vs. mean intensity diagram of $\eta \mathrm{UMa}$. 


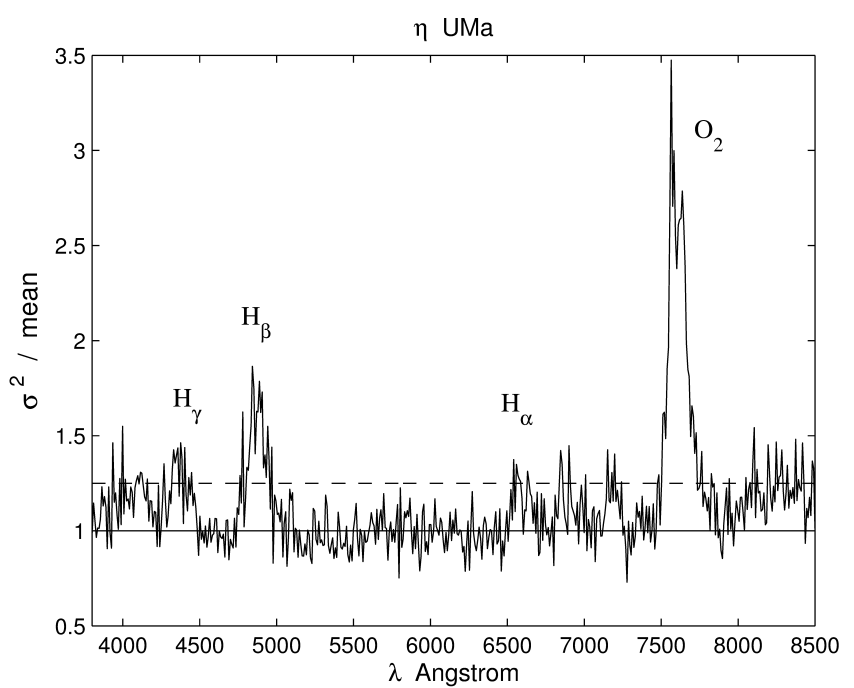

Fig. 7: Variations in the spectrum of $\eta \mathrm{UMa}$.

The absorption spectrum of $\eta \mathrm{UMa}$ in Fig. 5 is typical of that of B-type stars. Absorption lines of hydrogen and the atmospheric oxygen line $\mathrm{O}_{2}$ $(\lambda=7605 \AA)$ are present in the spectrum. The halfwidth line $\mathrm{H} \beta(\lambda=4861 \AA)$ corresponds to a spectral resolution of the spectrograph and is FWHM $=40 \AA$.

In measurements with CCD detectors, the readings (ADU) are proportional to the intensity and the gain. Fig. 6 shows the ratio of the variance to the mean intensity for the program star. The amplification factor is equal to two. The figure shows that the intensity follows the Poisson distribution (dispersion and average intensity are equal with an accuracy of approximately $1 \%$ ).

The following Fig. 7 shows the dependence of the variance to the mean intensity in the spectrum of the program star. For Poisson random variables, the following relation holds [6]:

$$
(n-1) \sigma^{2} / \operatorname{mean}=\chi^{2}
$$

where $n$ is the number of measurements. The formula allows setting a detection threshold by using the $\chi^{2}$ probability distribution. For $n=200$ the $99 \%$ detection threshold is 1.25 .

Fig. 7 shows that the hydrogen Balmer lines and atmospheric lines around $6800 \AA, 7300 \AA$ and $7605 \AA$ are variable. It follows that the variations in hydrogen lines comprise approximately from $0.2 \%$ to $0.5 \%$, while that of the oxygen line comprises up to $2 \%$.

Fig. 8 shows a similar relationship for the comparison star $77 \mathrm{Cyg}$. The $99 \%$ detection threshold for $n=500$ is 1.15 . The number of noise peaks above

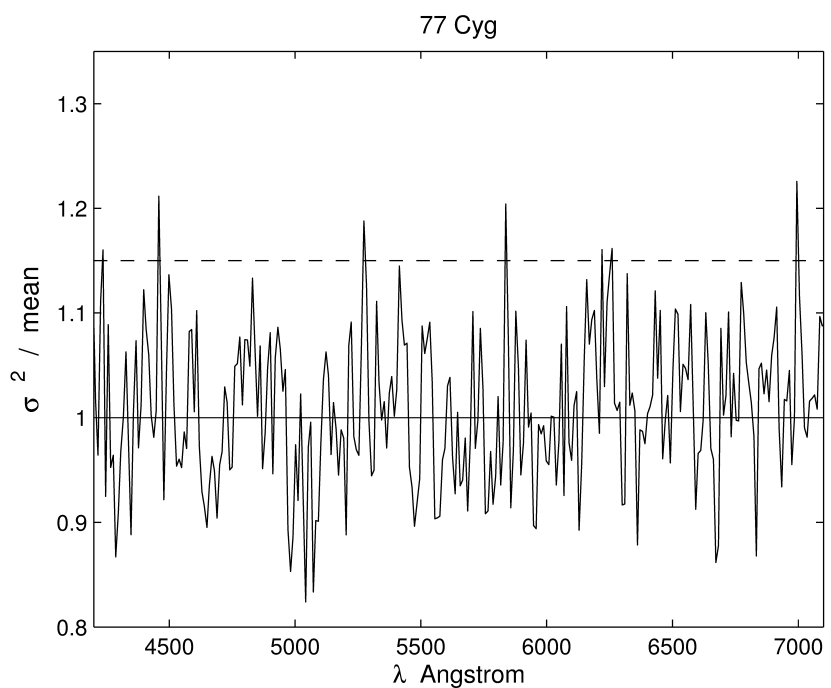

Fig. 8: Variations in the spectrum of $77 \mathrm{Cyg}$.

the detection threshold does not exceed $1.4 \%$. With $99 \%$ confidence it can be argued that the comparison star $77 \mathrm{Cyg}$ is not variable.

\section{CONCLUSIONS}

Dynamic spectroscopy of the hot star $\eta \mathrm{UMa}$ of spectral class B3V with a slitless spectrograph with a spectral resolution $\mathrm{R} \sim 100$ allowed us to detect rapid variations in hydrogen lines $\mathrm{H} \alpha, \mathrm{H} \beta, \mathrm{H} \gamma$ from $0.2 \%$ to $0.5 \%$, as well as variations in atmospheric absorption lines. The characteristic time of variation is in the sub-second range. Such rapid variations in the spectra of stars of the main sequence B is observed, apparently, for the first time. The mechanism of variations is unknown. Perhaps this is due to the stellar wind, which in stars of this class can reach up to $3000 \mathrm{~km} / \mathrm{s}$.

\section{REFERENCES}

[1] Smith M. A. 2006, A\&A, 459, 215

[2] Chojnowski S. D., Whelan D. G., Wisniewski J. P. et al. 2015, AJ, 149, 7

[3] Kukarkin B. V., Parenago P. P., Efremov Yu. N. \& Kholopov P.N. 1951, 'Catalogue of suspected variable stars', Acad. of Sciences USSR Sternberg, Moscow

[4] Cassinelli J.P., Cohen D.H., Macfarlane J. J., Sanders W. T. \& Welsh B. Y. 1994, ApJ, 421, 705

[5] Kaiser J. F. \& Reed W. A. 1977, Rev. Sci. Instruments, 48, 1447

[6] Pollard J. H. 1979, 'A handbook of numerical and statistical techniques', Cambridge University Press, Cambridge

[7] Zhilyaev B. E., Romanyuk Ya. O., Svyatogorov O. A. et al. 2007, A\&A, 465, 235 